\title{
Fine Mapping and Analysis of Candidate Genes for qFT7.1, a Major Quantitative Trait Locus Controlling Flowering Time in Brassica Rapa L
}

\section{Gaoyang Qu}

Shenyang Agricultural University College of Horticulture

\section{Yue Gao}

Shenyang Agricultural University

\section{Xian Wang}

Shenyang Agricultural University College of Horticulture

Wei Fu

Shenyang Agricultural University College of Horticulture

Yunxia Sun

Shenyang Agricultural University College of Horticulture

Xu Gao

Shenyang Agricultural University College of Horticulture

Wei Wang

Shenyang Agricultural University College of Horticulture

\section{Chunming Hao}

Shenyang Agricultural University College of Horticulture

Hui Feng

Shenyang Agricultural University College of Horticulture

Yugang Wang ( $\nabla$ ygwang@syau.edu.cn )

Shenyang Agricultural University College of Horticulture https://orcid.org/0000-0002-9417-513X

\section{Research Article}

Keywords: Flowering time, Brassica rapa, QTL, Fine mapping, Chromosome Segment Substitution Line

Posted Date: December 2nd, 2021

DOI: https://doi.org/10.21203/rs.3.rs-1103395/v1

License: (c) (1) This work is licensed under a Creative Commons Attribution 4.0 International License.

Read Full License 


\section{Abstract}

In Brassica rapa, flowering time (FT) is an important agronomic trait that affects the yield, quality, and adaption. FT a complicated trait that is regulated by many genes and is affected greatly by the environment. In this study, a chromosome segment substitution line (CSSL), CSSL16, was selected that showed later flowering than the recurrent parent, rapid-cycling inbred line of $B$. rapa (RcBr). Using Bulked Segregant RNA sequencing, we identified a late flowering quantitative trait locus (QTL), designated as qFT7.1, on chromosome $A 07$ based on a secondary- $\mathrm{F}_{2}$ population derived from the cross between CSSL16 and RcBr. qFT7.1 was further validated by conventional QTL mapping. This QTL explained $39.9 \%$ (logarithm of odds $=32.2$ ) of the phenotypic variations and was fine mapped to a 56.4-kb interval using recombinant analysis. Expression analysis suggests that BraA07g018240.3C, which is homologous with ATC (encoding Arabidopsis thaliana CENTRORADIALIS homologue), a gene for delayed flowering in Arabidopsis as the most promising candidate gene. Sequence analysis demonstrated that two synonymous mutations existed in the coding region and numerous bases replacements existed in promoter region between BraA07g018240.3C from CSSL16 and RcBr. The results will increase our knowledge related to the molecular mechanism of late flowering in $B$. rapa, and lay a solid foundation for the breeding of late bolting in B. rapa.

\section{Key Message}

qFT7.1, a major QTL for flowering time in Brassica rapa was fine-mapped to chromosome A07 in a 56.4$\mathrm{kb}$ interval in which the most likely candidate gene is BraA07g018240.3C.

\section{Introduction}

The economically important crop, Brassica rapa, has long been cultivated worldwide, mainly as a vegetable foodstuff, such as Chinese cabbage and Pak-choi, and to a lesser extent for the production of fodder and oilseed, such as turnip rape and yellow sarson (Carpio et al. 2011). Among agronomic traits in B. rapa, flowering time (FT) is important because it affects the yield of seeds and the harvested crop's commercial quality (Wu et al. 2012), thus determining their growing season and cultivation area (Xiao et al. 2019). The regulation of FT is complex, involving multiple genes (quantitative trait loci (QTLs)) and is markedly affected by environmental conditions, making it a challenge to identify linked markers or related genes for marker assisted selection (MAS)-based breeding (Liu et al. 2016).

Many QTLs related to FT in B. rapa have been identified in the last 30 years, mainly based on a wide range of bi-parental mapping populations or natural populations (Teutonico and Osborn 1995; Osborn et al. 1997; Ajisaka et al. 2001; Schranz et al. 2002; Nishioka et al. 2005; Yang et al. 2007; Lou et al. 2007; Li et al. 2009a; Yuan et al. 2009; Zhao et al. 2010; Lou et al. 2011, Kakizaki et al. 2011; Xiao et al. 2013; Li et al. 2015; Zhang et al. 2015; Liu et al. 2016; Xi et al. 2018; Wang et al. 2018b; Xiao et al. 2019; Fu et al. 2020; Kaur et al. 2021). The majority of these populations are traditional primary populations, including $\mathrm{F}_{2}, \mathrm{BC}_{1}$, doubled haploid (DH), and recombinant inbred lines (RILs). However, these populations are only 
useful to detect QTLs with relatively large effects, because the segregants often have complicated backgrounds due to the presence of large parent-derived chromosomal fragments, thus QTLs with minor effects might be missed. By contrast, a wider range of QTLs can be identified using advanced mapping populations, including nearisogenic lines (NILs) and chromosome segment substitution lines (CSSLs) (Nadean and Frankel. 2000). Moreover, secondary $F_{2}$ or $F_{3}$ populations can be produced by from backcrossing a target NIL or CSSL with the recurrent parent, making them more suitable for fine mapping and positional cloning of a target QTL (Yano. 2001). Over 75 CSSL libraries in 17 major crops have been constructed in the last three decades, which have made significant contributions to QTL characterization (Balakrishnan et al. 2019), despite the development of these population being labor and time intensive. However, currently, only few CSSLs are available for B. rapa (Li et al. 2015; Wang et al. 2018b). Most of the above mentioned QTLs identified in primary populations have not yet to be fine mapped, mainly because of a lack of optimal genetic material, and very few studies resulted in the actual cloning of the gene responsible for flowering in $B$. rapa. To the best of our knowledge, to date, only a few genes, such as BrVIN3.1, BrFLC1, BrFLC2 (Su et al. 2018; Jeong et al. 2019) and BraELF6 (Li et al. 2019) have been successfully cloned and subsequently confirmed by transformation in B. rapa and in Arabidopsis thaliana, respectively.

NILS and CSSLs have been demonstrated as effective resources to validate QTLs and can be used to create fine-mapping populations (Fletcher et al. 2013). CSSLs (or NILs) lines containing a single (or several) genomic introgression from the donor parent in a different, but homogenous, genomic background. Thus, CSSLs eradicate the 'noisy' genetic background, permitting QTLs to be resolved as Mendelian factors. CSSLs and NILs have advantages in transcriptional analyses because interference from the genetic background is minimized, thus enhancing the accuracy and sensitivity of transcriptional analysis (Keurentjes et al. 2007). Transcriptional analysis (e.g., RNA sequencing (RNA-seq)) based on CSSLs (or NILs) has contributed to the rapid identification of candidate genes underlying QTLs, especially in species with large polyploid genomes, such as wheat (Barrero et al. 2015; Xiao et al. 2016; Habib et al. 2018; Yang et al. 2018; Jiang et al. 2019).

Recently, the identification and isolation of genes underpinning QTLs associated with agronomic traits in crops have been accelerated significantly because of the emergence of rapid and inexpensive nextgeneration sequencing (NGS)-based technologies combined with plant genetics (Nguyen et al. 2019). An example of such achievement is QTL-seq (Takagi et al. 2013), which combined with bulk-segregant analysis (BSA), can rapidly discover associated markers and candidate genes by sequencing the bulks and parents with extreme phenotypes from the segregating populations, using BSA based on DNA-and RNA-seq (Zou et al. 2016). Traditional QTL mapping integrated or combined with QTL-seq and RNA-seq is a highly efficient and accurate approach for QTL mapping and validation, which enables the identification of candidate genes associated with agronomic traits of interest, and has been widely utilized in diverse crops (Lu et al. 2014; Berenguer et al. 2015; Gelli et al. 2017; Li et al. 2017; Shu et al. 2018; Wang et al. 2018a; Liu et al. 2019; Park et al. 2019; Wen et al. 2019; Huang et al. 2021). 
Previously, a set of CSSLs was developed by our group using rapid-cycling inbred line of Brassica rapa $(\mathrm{RcBr})$ and the Chinese cabbage inbred line $08 \mathrm{A061}$ as the recipient and donor parents, respectively (Wang et al. 2018b). Among the developed CSSLs, CSSL16 showed significantly later flowering than the recurrent parent, $\mathrm{RcBr}$. To map and identify the candidate gene(s) responsible for this late flowering, we developed a secondary $F_{2}$ population derived from a cross between CSSL16 and RcBr. Bulked Segregant RNA-Seq (BSR-Seq) identified a QTL, qFT7.1, which was validated using conventional QTL mapping. Ultimately, recombinant analysis narrowed down $q F T 7.1$ to a $56.4-\mathrm{kb}$ interval on chromosome A07, allowing the candidate genes to be identified. Our findings represent a benchmark to further determine the molecular mechanism of late flowering in B. rapa.

\section{Materials And Methods}

\section{Plant materials and trait measurement}

Our laboratory previous constructed a set of CSSLs populations from a cross between $\mathrm{RcBr}$ as the recipient parent, which is an extremely early-flowering and vernalization independent inbred line, and 08A061 as the donor parent, which is a very late-flowering and vernalization dependent Chinese cabbage inbred line (Wang et al. 2018b). In the CSSL population, one line, CSSL16, with a late flowering phenotype, was chosen for backcrossing with the recurrent parent, $\mathrm{RcBr}$. The secondary $\mathrm{F}_{2}$ CSSL16/RcBr population was subsequently constructed using self-pollination.

The phenotypic analyses were carried out at the Experiment Station of Shenyang Agriculture University, Shenyang, China $\left(41.8^{\circ} \mathrm{N}, 123.4^{\circ} \mathrm{E}\right)$. Four flowering indices were assessed to calculate the phenotypic scores of the individuals: The bolting index $(\mathrm{BI})$, days to reach a $5 / 10-\mathrm{cm}$-high elongated floral stalk (DE5/DE10), and FT (Liu et al. 2016). CSSL16 and RcBr were evaluated under four environmental conditions (E1, E2, E3 and E4, Table S1). A secondary $F_{2}$ population consisted of 500 individuals was sown in the green house in September 2019, which were utilized for BSR-Seq and 300 individuals were used for conventional QTL analysis in March 2020. The progeny of the recombinant individuals screened from the secondary $F_{2}$ population (2200 individuals), along with parental controls, were grown in March 2021 for fine mapping of the identified QTL. All plants were sown directly into 10-cm pots without providing any extra vernalization, as described in our previous study (Wang et al. 2018b).

\section{RNA isolation and extreme pool construction}

For BSR-Seq analysis, we constructed two extreme pools, a L-pool (late-flowering pool) and an E-pool (early flowering pool), by mixing the same amounts of RNA from 25 late-flowering or 25 early-flowering plants, according to the phenotypic scores of $500 \mathrm{~F}_{2}$ individuals recorded in the fall of 2019. About 50 days after sowing, the apex leaves of each pool were sampled and subjected to RNA. The TRIzol reagent (Invitrogen, Carlsbad, CA, USA) was used to extract the total RNA. An Agilent 2100 Bioanalyzer (Agilent Technologies, Palo Alto, CA, USA), a NanoDrop spectrophotometer (Thermo Fisher Scientific Inc., Waltham, MA, USA), and $1 \%$ agarose gel were used to assess the quantity and quality of the extracted 
RNA. RNA $(1 \mu \mathrm{g})$ with an RNA integrity number $(\mathrm{RIN})>7$ was then processed for next generation sequencing library construction (NEBNext ${ }^{\circledR}$ Ultra $^{\text {TM }}$ RNA Library Prep Kit for Illumina ${ }^{\circledR}, N E B$, Ipswich, MA, USA).

\section{BSR-seq}

The prepared cDNA libraries were sequenced using the Illumina HiSeq 2500 platform (Illumina, San Diego, CA, USA) in the 2'150 bp paired-end (PE) configuration, after which the sequences were processed and analyzed by GENEWIZ (Suzhou, China). To remove technical sequences, including adapters, polymerase chain reaction (PCR) primers (or fragments thereof) and bases with quality lower than 20 , Trimmomatic (v0.30) (Bolger et al. 2014) was used to generate high quality clean data. The clean data were mapped to the $B$. rapa reference genome V3.0 (BRAD, http://brassicadb.cn/\#/Download/) using Hisat2 (Zhang et al. 2018a). Samtools v0.1.18 (Li et al. 2009b), with the command mpileup, and Bcftools v0.1.19 (Narasimhan et al. 2016) were used to carry out Single Nucleotide Variation (SNV) calling. The Euclidean distance (ED) value was calculated based an mpileup file which was generated using samtools v0.1.18 for BSR-seq.

According to the basic principle of the ED value, the occurrence frequency of the four bases $A, T, C$ and $G$ at the SNV site was statistically different in the population, and the corresponding base frequency of the two trait groups was calculated by distance. To eliminate the background noise, the ED value of each different SNV site was raised to the power of five, termed ED ${ }^{5}$ (Su et al. 2016). All ED ${ }^{5}$ values were sorted, and the different SNV sites corresponding to the top $1 \%$ of ED ${ }^{5}$ values were screened, and then specific chromosome segments were located according to the distribution of the different SNV sites.

\section{DNA extraction and marker development}

The CTAB method, with minor modifications (Murray and Thompson 1980), was used to extract total DNA from the two parental lines and individuals of the secondary $F_{2}$ populations. The PCR reaction volume and amplification were same as those described previously (Wang et al., 2018b). The markers were designed using Primer Premier 5.0 software (Premier Biosoft, San Francisco, CA, USA) based on sequence variations of the target region identified by BSR-seq and whole-genome re-sequencing between $\mathrm{RcBr}$ and CSSL16. The primer information is shown in Table S2.

\section{QTL analysis and fine mapping}

The BSR-seq-identified QTL for FT was confirmed using classical QTL mapping assisted by polymorphic markers. The secondary $F_{2}$ populations utilized for conventional QTL analysis consisted of 300 individuals sown in March 2020. QTL mapping was performed using composite interval mapping (CIM) implemented in Windows QTL Cartographer 2.5 (Silva et al. 2012). The determined logarithm of odds (LOD) value for putative QTL declaration was determined after 1000 permutation tests at a significance level of $P<0.05$ and a threshold of 3.0 . 
The progeny of recombinant individuals screened from a larger secondary $F_{2}$ population $(2200$ individuals) sown in a green house in March 2021 was used for fine mapping of the identified QTL. The means of the homozygous recombinant phenotype of the progeny were analyzed using Student's $t$-test in SPSS v17.0 (IBM Corp., Armonk, NY, USA), and compared with that of the control (RcBr) at a significance level of $P<0.01$.

\section{Whole-genome resequencing}

Total DNA was extracted from young leaves. Its quality was determined using $0.8 \%$ agarose gel electrophoresis and it was quantified using an ultraviolet spectrophotometer. The Illumina NovaSeq platform was used for 2'150 bp paired-end sequencing. The raw data were was cleaned using Adapter Removal (version 2) (Schubert et al. 2016), and high quality reads were compared with the Brassica rapa V3.0 reference genome. Single nucleotide polymorphisms (SNPs) and InDels were detected by GATK software (Van et al. 2013) and analyzed using the ANNOVAR software (McKenna et al. 2010, Wang et al. 2010).

\section{Candidate gene sequence analysis}

The annotation information of genes in the candidate region was obtained from the $B$. rapa database (BRAD, http://brassicadb.cn/\#/Download/) and The ArabidopsisInformation Resource (TAIR, http://www.arabidopsis.org/). The specific primers (Table S2) to amplify the full-length coding sequences and promoter sequences were designed using Primer 5.0 (Premier Biosoft). A Gel Extraction Kit (CWBIO, Beijing, China) was used to purify the PCR products, which were ligated into a pGEM-T Easy Vector (Promega, Madison, WI, USA), followed by sequencing at GENEWIZ. The sequences were aligned using the DNAMAN software (Lynnon Biosoft, San Ramon, CA, USA) and the structure of the candidate gene was displayed using online software (http://gsds.cbi.pku.edu.cn/).

\section{Quantitative real-time reverse transcription PCR (qRT-PCR) analysis of candidate gene expression}

The expression level of the candidate gene was detected using qRT-PCR. Total RNA of RcBr and CSSL16 from roots, leaves, cotyledons, hypocotyls, stems, flowers, and the shoot apical meristem (SAM) were isolated using an RNA extraction kit (Aidlab Biotechnologies Co., Ltd., Beijing, China). The RNA was then reverse transcribed to CDNA. The CDNA was then used as the template for the qRT-PCR reaction (reaction volume: $25 \mu \mathrm{L}$, comprising $2 \times$ Ultra SYBR Mixture, $2 \mu \mathrm{L}$ of diluted cDNA, $1 \mu \mathrm{L}$ of $0.2 \mu \mathrm{M}$ forward and reverse primers, and $21 \mu \mathrm{L}$ of RNase-free water). The reaction conditions were: initial denaturation at 95 ${ }^{\circ} \mathrm{C}$ for $10 \mathrm{~min}$, followed by 40 cycles of $95^{\circ} \mathrm{C}$ for $15 \mathrm{~s}$ and $60^{\circ} \mathrm{C}$ for $1 \mathrm{~min}$. This was followed by meltingcurve analysis: $95^{\circ} \mathrm{C}$ for $15 \mathrm{~s}, 60^{\circ} \mathrm{C}$ for $1 \mathrm{~min}, 95^{\circ} \mathrm{C}$ for $15 \mathrm{~s}$, and $60^{\circ} \mathrm{C}$ for $15 \mathrm{~s}$. The $2^{-} \Delta \Delta \mathrm{Ct}$ method was used to analysis the relative expression level. Cycle threshold $(\mathrm{Ct})$ values were shown as the means of three independent biological replicates. Each sample was analyzed as three independent technical replicates. QuantStudio ${ }^{\text {TM}} 6$ Flex Manager software (Livak and Schmittgen 2001) was used to analyze the data. Primer 5.0 was used to design gene-specific primers (shown in Table S2), with the Actin gene being used as the internal control (Huang et al. 2015). 


\section{Result}

\section{Genotypic and phenotypic characterization of RcBr and CSSL16}

To detect the segment which had introgressed from 08A061 on ten chromosomes of CSSL16, the two parental lines, $\mathrm{RcBr}$ and CSSL16, were genotyped using whole-genome re-sequencing. A total of $67,507,156$ and $64,540,134$ high quality reads were detected in $\mathrm{RcBr}$ and CSSL16, and the clean data was compared to reference genome. This identified 2,999,421 SNPs and 627,670 InDels on the ten chromosomes between the two parental lines. According to the ED value calculation, the variation was mainly distributed in chromosome A02 (physical location 1,775,235-2,512,196 bp) and A07 (physical location 15,350,379-16,648,887 bp) (Fig.1). The total substituted segment derived from the donor parent, $08 \mathrm{~A} 061$ was approximately $2.04 \mathrm{Mb}$, and the $B$. rapa whole genome size is about $353.14 \mathrm{Mb}$ (B. rapa V3.0), and the background recovery rate was about $99.42 \%(351.10 / 353.14)$.

$\mathrm{RcBr}$ and CSSL16 showed a significant difference in FT under multiple environments (Fig.2a). Under E1 growth conditions, the FT of $\operatorname{RcBr}(75.47 \pm 3.78)$ was earlier than CSSL16 $(98.19 \pm 2.07)$ by about 23 days, under E2 conditions, the FT of $\operatorname{RcBr}(52.26 \pm 1.27)$ was earlier than CSSL16 (63.43 \pm 1.28$)$ by about 11 days, under E3 conditions, the FT of $\operatorname{RcBr}(44.21 \pm 3.55)$ was earlier than CSSL16 (61.69 \pm 3.88$)$ by about 17 days, and under E4 conditions, the FT of $\operatorname{RcBr}(38.78 \pm 2.78)$ was earlier than CSSL16 (51.55 \pm 3.37 ) by about 13 days (Fig.2b). The two parental lines also showed significant differences in DE5, DE10, and BI under all four growth conditions (Table 1). In conclusion, RcBr and CSSL16 showed significant difference in all flowering-related traits.

\section{Identification and validation of $q F T 7.1$}

Through BSR-seq analysis, we were able to map 45,425,180 and 41,697,006 clean reads to the $B$. rapa reference genome from the E-pool and L-pool, respectively. A total of 218,944 SNPs in the E-pool and 209,924 SNPs in the L-pool were identified. All ED ${ }^{5}$ values were sorted, the top $1 \%$ of ED ${ }^{5}$ values was used as the threshold and the different corresponding SNV sites were screened. The distribution of different SNV sites confirmed the candidate region. This candidate region of the QTL for FT was located on chromosome A07, starting at 15,486,952 and ending at 16,546,846, thus, the candidate interval covers about $1.06 \mathrm{Mb}$ (Fig. 3). The candidate QTL underlying FT in this region was designated as qFT7.1.

To validate the QTL, $q F T 7.1$, identified by BSR-seq analysis, we carried out conventional QTL analysis with $300 \mathrm{~F}_{2}$ individuals in March 2020. A total of 13 polymorphic markers (Table S2) were screened from the difference interval (donor segment of 08A061), which was detected using whole genome resequencing (Chromosomes A02 and A07), and all polymorphic markers were used for classical QTL mapping. One QTL with a LOD value of 32.2, explaining 39.9\% of the phenotypic variation, was found to control FT, and was located between marker InDel714 and InDel716, corresponding to a physical position of 15,539,588 bp to 16,499,043 bp on chromosome A07 (Fig. 4b), however, we could not detect any QTL on chromosome A02. Thus, the conventional QTL analysis confirmed the QTL qFT7.1, which was identified via BSR-seq analysis. 


\section{Fine mapping of $q F T 7.1$}

The candidate QTL, qFT7.1, was preliminary mapped to a $1.06 \mathrm{Mb}$ candidate region on chromosome A07. A larger $F_{2}$ population consisting of 2200 individuals was used to refine the position of qFT7.1. Recombinant plants were screened with markers InDel723 and InDel716 and a total of 19 recombinant individuals were obtained. The homozygous recombinant progeny were divided into ten groups according to their genotypes. The mean value of the homozygous progeny (DE5, DE10 and FT) were compared with $\mathrm{RcBr}$ at $P<0.01$ level.

Recombinants L1 and L2 both showed no difference with RcBr, while L3 and L4 had the opposite genotype to L1 and L2. Recombinants L3 and L4 both showed significant differences with RcBr, but no difference with CSSL16, thus these groups placed the QTL to a region upstream of markers InDel705 and InDel708. In the same way, the results for recombinants L5 and L6 demonstrated that the QTL was located upstream of markers InDel706 and InDel707, and the results for recombinant L7 placed the QTL in a region upstream of marker InDel702. Furthermore, recombinant L8 identified that the QTL was located upstream of marker InDel715, whereas recombinant L9 was significantly different from CSSL16 and similar to $\mathrm{RcBr}$, which delimited the QTL to a region downstream of marker InDel714. Recombinant group L10 showed a significant difference with RcBr, thus group L10 placed the QTL in a region downstream of marker InDel723. By positioning of the recombinant groups, qFT7.1 was finally narrowed down to a 56.4-kb interval between marker InDel714 and InDel715, and physical position of $15,539,588$ bp to $15,595,959$ bp on chromosome A07 (Fig. 4c).

\section{Candidate gene annotation}

According to the $B$. rapa reference genome database annotation, nine genes were annotated to the 56.4$\mathrm{kb}$ region (BraA07g018220.3C-BraA07g018300.3C). The detailed information for the genes is shown in Supplementary Table 3. All the genes were compared with Arabidopsishomolog genes and analyzed for their function, we found that BraA07g018240.3C is homologous with Arabidopsis gene At2g27550, a key gene regulating FT. This gene was an Arabidopsis CENTRORADIALIS homologue (ATC) gene which belongs to FLOWERING LOCUS T(FT)family and encodes a protein similar to TERMINAL FLOWER1 (TFL 1), the overexpression of which leads to a similar phenotype as TFL 1. The encoded protein from the identified gene might inhibit the expression of critical flowering genes LEAFY (LFY) and APETALA1 (AP1), acting in a non-cell autonomous manner to delay flowering (Huang et al. 2012, Zhu et al. 2020).

\section{Expression analysis by qRT-PCR}

The results indicated that $B r a A 07 g 018240.3 \mathrm{C}$ was specifically expressed in the root and hypocotyl, and not in other tissues. The expression level showed significant differences in the hypocotyl, but not in the root between $\mathrm{RcBr}$ and CSSL16 (Fig. 5). The expression of the others eight genes was also detected in the root, stem, leaf, and flower, with BraA07g018270.3C and BraA07g018300.3C showing significantly different expression levels in flower (Fig. 6). 
The signals of each flowering pathway were collected in the SAM, and were used together to determine the FT. $A P 1$ and $L F Y$ are both main inflorescence meristem genes, and play a central role in the flowering regulatory network (Wellmer and Riechmann 2010). To verify the most likely candidate gene, the expression of $\angle F Y$ and $A P 1$ homologous genes in the SAM were detected in $\mathrm{RcBr}$ and CSSL16. $L F Y$ homologous genes included BraA02g043220.3C and BraA06g025360.3C, and the AP1 homologous gene included BraA02g018970.3C, BraA07g030470.3C, and BraA07g034100.3C in B. rapa. Five specific primers, RT-22, RT-36, RT-97, RT-47, and RT-41 were used to analyze the expression levels of the $A P 1$ and $L F Y$ genes (Table S2). The results showed that the expression levels of all the $L F Y$ and $A P 1$ homologous genes were significantly different between $\mathrm{RcBr}$ and CSSL16, with all the genes being downregulated in CSSL16 (Fig. 7). Higher expression of $\angle F Y$ and $A P 1$ result in Arabidopsis premature flowering, therefore, ATC (BraA07g018240.3C) possibly downregulates the expression of $L F Y$ and $A P 1$, which would lead to delayed flowering, similar to the function of $A T C$ in $A$. thaliana. In conclusion, we predicted that BraA07g018240.3C was the most likely candidate gene. To further confirm the candidate gene, we analyzed the sequence variations of $B r a A 07 g 018240.3 \mathrm{C}$ between the two parental lines.

\section{Sequence variation analysis of BraA07g018240.3C}

To identify variations in the candidate gene sequence, a specific primer, 24-C, was used to detect CDS sequence variation (Table S2). The full length gene for BraA07g018240.3C is $1600 \mathrm{bp}$, starting at $15,558,430$ and ending at 15,560,029, including three introns and four exons. The CDS sequence had an A to $T$ mutation at the $12^{\text {th }}$ base in first exon and a base $T$ to $C$ change at the $32^{\text {nd }}$ base in the second exon, however, mutations were both synonymous (Fig. 4d). Two specific primers, QG-1 and QG-22 (Table S2), were used to amplify the promoter. The result showed many changes in the promoter regions of BraA07g018240.3Cbetween two parental lines (Fig. S1).

\section{Discussion}

In this study, we employed BSR-seq based on secondary $F_{2}$ populations derived from CSSL 16 and $R c B r$ to identify the QTL qFT7.1 (Fig. 3), which is responsible for late flowering in B. rapa, and was further validated using classical QTL mapping (Fig. 4c). QTL-seq (BSR-seq) combined with classical QTL mapping has proven to be a powerful tool to identify major QTLs controlling traits of interest in a variety of crops (Lu et al. 2014; Berenguer et al. 2015; Gelli et al. 2017; Shu et al. 2018; Park et al. 2019; Wen et al. 2019; Huang et al. 2021). Thus, most of the populations utilized for QTL-seq are preliminary populations, such as $F_{2}$, DHs, and RILs. QTL-seq utilized mainly for detecting major QTLs with large effects, QTLs with minor effects might not be detected by QTL-Seq, as traditional QTL mapping did. Recently, Zhang et al. (2019) devised a new method, quantitative trait gene sequencing (QTGseq), to accelerate QTL fine mapping through QTL partitioning and wholegenome sequencing of BSA populations. QTL partitioning is a strategy used to convert a quantitative trait into a near-qualitative trait quickly in nature. However, compared with the line developed by QTG-seq, CSSLs (or NILs) showed a relatively homozygous genetic background, only the target region is a substitution fragment, and other regions are not replaced; therefore, the background is basically the same as the recurrent parent, and the positioning accuracy was 
relatively high. Furthermore, transcriptional analysis (RNA-seq) based on CSSLs (or NILs) also has advantages for identifying candidate genes underlying QTLs (Keurentjes et al. 2007).

Ultimately, qFT7.1 was fine mapped to a 56.4-kb interval, between the two InDel markers, InDel714 and InDel715, (Fig. 4C) and a physical position of 15,539,588 to 15,595,959 on chromosome A07. In our previous studies, we did not detected any QTLs in the candidate region basing on $F_{2}$, RIL, and CSSLS derived from the identical parent, RcBr and 08A061 (Wang et al. 2014; Liu et al. 2016; Wang et al. 2018b). The CSSLs were constructed using $166 \mathrm{InDel}$ and SSR markers that were distributed relatively evenly on the ten chromosomes; however, a low marker density is likely to lead to small introgression segments being missed. We did not detect any introgression segments on chromosome A07 for CSSL16 based on a limited number of markers (data not shown); therefore, we re-sequenced the two parental lines to identify the possible segment derived from 08A061 (Fig. 1). Until now, no other flowering related QTLs were detected in the candidate interval of qFT7.1 on chromosome A07 in B. rapa, allowing us to identify the Arabidopsis ATC homologous gene for the first time, which is of a great significance to breed late flowering varieties of $B$. rapa.

ATC is a TFL 1-like gene in Arabidopsis that is homologous with CENTRORADIALIS (CEN) and was first identified in Antirrhinum (Bradley et al. 1996). ATC inhibits flowering and regulates inflorescence morphology (Banfield and Brady 2000). ATC encodes a protein that is $77 \%$ similar to Antirrhinum CEN and $67 \%$ similar to TFL 1 at the amino acid level. ATC, CEN, and TFL 1 overexpression showed similar phenotypes in wild-type Arabidopsis (Ratcliffe et al. 1998; Mimida et al. 2001). ATC acts in a non-cell autonomous manner to inhibits flowering in Arabidopsis, and is specifically expressed in the hypocotyl, through long distance movement to the SAM. $L F Y$ and $A P 1$ are the critical factors that regulate FT positively in the SAM. ATC might inhibit the expression of $L F Y$ and $A P 1$ to delay flowering (Hempel et al. 2000; Huang et al. 2012; Gao et al. 2017). Many ATC homologous genes have been identified in different species, such as GoCEN-Dt in cotton, Hordeum vulgare CENTRORADIALIS (HVCEN) in barley, SELFPRUNING (SP) in tomato, and ZEA CENTRORADIALIS (ZCN) in maize (Pnueli et al. 1998; Danilevskaya et al. 2010; Coradran et al. 2012; Liu et al. 2018). These studies indicated that ATC homologous proteins have similar functions and their upregulated expression delays flowering. Our study also found that the BraA07g018240.3C expression level in CSSL16 was higher than that in RcBr, which fits with the prediction that upregulated expression of this ATC-like gene would delay the flowering; however, this function needs to be verified in transgenic plants.

BraA07g018240.3C was homologous with Arabidopsis gene ATC (At2g27550), which belongs to the FT family and acts systemically to inhibit floral initiation. We found that BraA07g018240.3C was expressed specifically in the hypocotyl and root, which was consistent with the results of Huang et al. (2012), in Arabidopsis, the ATC gene is mainly expressed in vascular tissues, but not in the apex. In the present study, we could not detect any expression (the number of reads was zero) of BraA07g018240.3C based on BSR-seq, which proved that $B r a A 07 g 018240.3 C$ is a specially expressed gene in $B$. rapa. According to our results, the expression of $A P 1$ and $\angle F Y$ genes in the SAM was significantly higher in $\mathrm{RcBr}$ than in CSSL16, which, consisted with the results reported by Liu et al (2009) and Kaneko-Suzuki et al. (2018), 
allowed us to speculate that $A T C$ might inhibit $A P 1$ and $L F Y$ expression positively by long distance transport in the SAM, which then delays flowering in B. rapa.

We cloned the candidate gene BraA07g018240.3C promoter and CDS sequence, and identified two synonymous mutations in the CDS and some mutated bases in the promoter sequence. Changes in the promoter region might influence gene expression, for example, tomato SELF PRUNING $5 G$ is a critical gene for FT, and Zhang et al (2018b) found that changes to the promoter region resulted in delayed flowering. The candidate gene for a major QTL controlling tomato weight, fw2.2, also had changes in its promoter sequence, which influenced fruit weight (Nesbitt and Tanksley. 2002). In B. rapa, Su et al (2021) identified sequence variations in the promoter of BrHISN2, which conferred cold-dependent expression on $B r H I S N 2$, resulting in leaf yellowing. Thus, the significantly different expression levels of BraA07g018240.3C between the two parental lines might be caused by changes in the promoter region.

In conclusion, we fine mapped a novel QTL for FT, $q F T 7.1$, to a physical interval of $56.4 \mathrm{~kb}$ on chromosome A07. The CSSL16 allele at the qFT7.1 locus regulates the FT negatively at the bolting stage of $B$. rapa. According to the sequence and expression level, the most likely candidate gene, BraA07g018240.3C, encodes a TFL 1-like protein. These findings could aid our understanding of the mechanisms underlying flower formation and provide a genetic resource for $B$. rapa crop improvement studies.

\section{Declarations}

\section{Author contribution statement}

YW designed the experiments. YG, XW, YS, XG, WW and $\mathrm{CH}$ helped developing the CSSLs. GQ conducted the experiments, wrote the manuscript and performed the data analysis, HF and WF assisted in the data analysis. GQ and YW revised the manuscript. All authors reviewed and approved this submission. The authors note that this research was performed and reported in accordance with ethical standards of the scientific conduct.

\section{Acknowledgments}

The research was supported by the National Natural Science Foundation of China (Grant No. 32072569) and the Provincial Natural Science Foundation of Liaoning, China (Grant No. LSNJC202006). We would like to thank Elixigen for English language editing.

Code availability Not applicable, software used according to manuals.

\section{Ethical statement}

The authors note that this research was performed and reported in accordance with ethical standards of the scientific conduct. 


\section{Conflicts of interest}

The authors declare that they have no conflicts of interest.

\section{Availability of data and material}

The datasets generated and analyzed during this study are available on reasonable requests from the corresponding authors.

Consent to participate Not applicable.

Consent for publication Not applicable.

Open Access This article is licensed under a Creative Commons Attribution 4.0 International License, which permits use, sharing, adaptation, distribution and reproduction in any medium or format, as long as you give appropriate credit to the original author(s) and the source, provide a link to the Creative Commons licence, and indicate if changes were made. The images or other third party material in this article are included in the article's Creative Commons licence, unless indicated otherwise in a credit line to the material. If material is not included in the article's Creative Commons licence and your intended use is not permitted by statutory regulation or exceeds the permitted use, you will need to obtain permission directly from the copyright holder. To view a copy of this licence, visit http://creativecommons.org/licenses/by/4.0/.

\section{References}

1. Ajisaka H, Kuginuki Y, Yui S, Enomoto S and Hirai M (2001) Identification and mapping of a quantitative trait locus controlling extreme late bolting in Chinese cabbage (Brassica rapa L. ssp. Pekinensis syn. Campestris L.) using bulked segregant analysis. Euphytica 118:75-81. https://doi.org/10.1023/A:1004023532005

2. Balakrishnan D, Surapaneni M, Mesapogu S, Neelamraju S (2019) Development and use of chromosome segment substitution lines as a genetic resource for crop improvement. Theor Appl Genet 132:1-25. https://doi.org/10.1007/s00122-018-3219-y

3. Banfield MJ, Brady RL (2000) The structure of Antirrhinum centroradialis protein (CEN) suggests a role as a kinase regulator. J Mol Biol 297:1159-1170. https://doi.org/10.1006/jmbi.2000. 3619

4. Barrero JM, Cavanagh C, Verblya KL, Tibbts JFG, Verbyla AP, Huang BE, Rosewarne GM, Stephen S, Wang PH, Whan A, Rigault P, Hayden MJ, Gubler F (2015) Transcriptomic analysis of wheat nearisogenic lines identifies $P M 19-A 1$ and $A 2$ as candidate for a major dormancy QTL. Genome Biol 16:93. https://doi.org/10.1186/s13059-015-0665-6

5. Berenguer El, Houten JV, Huang ZJ, Knaap EVD (2015) Rapid and reliable identification of tomato fruit weight and locule number loci by QTL-seq. Theor Appl Genet 128:1329-1342. https://doi.org/10.1007/s00122-015-2509-x 
6. Bolger AM, Lohse M, and Usadel B (2014) Trimmomatic: a flexible trimmer for Illumina sequence data. Bioinformatics 30:2114-2120. https://doi.org/10.1093/bioinformatics/btu170

7. Bradley D, Carpenter R, Copsey L, Vincent C, Rothstein S, Coen E (1996) Control of inflorescence architecture in Antirrhinum. Nature 379:791-797. https://doi.org/10.1038/ 379791a0

8. Carpio DP, Basnet PK, De Vos RCH, Maliepaard C, Visser C, Bonnema G (2011) The patterns of population differentiation in a Brassica rapa core collection. Theor Appl Genet 122:1105-1118. https://doi.org/10.1007/s00122-010-1516-1

9. Comadran J, Kilian B, Russell J, Ramsay L, Stein N, Ganal M, Marshall D, Hedley P, Tondelli A, Peccchioni N, Francia E, Korzun V, Walther A, Waugh R (2012) Natural variation in homolog of Antirrhinum CENTRORADIALIS contributed to spring growth habit and environmental adaption in cultivated barley. Nat Genet 44:1388-1392. https://doi.org/ 10.1038/ng.2447

10. Danilevskaya ON, Meng X, Ananiev EV (2010) Concerted modification of flowering time and inflorescence architecture by ectopic expression of TFL 7-like genes in maize. Plant Physiol 153:238251. https://doi.org/10.1104/pp.110.154211

11. Fletcher RS, Mullen JL, Yoder S, Bauerle WL, Reuning G, Sen S, Meyer E, Juenger TE, and McKay JK (2013) Development of a next-generation NIL library in Arabidopsis thaliana for dissecting complex traits. BMC genomics 14:655. https://doi.org/10.1186/1471-2164-14-655

12. Fu W, Huang S, Gao Y, Zhang M, Qu G, Wang N, Liu Z, Feng H (2020) Role of BrSDG8 on bolting in Chinese cabbage (Brassica rapa). Theor Appl Genet 133:2937-2948. https://doi.org/10.1007/s00122-020-03647-4

13. Gao J, Huang BH, Wan YT, Chang J, Li JQ, Liao PC (2017) Functional divergence and intron variability during evolution of angiosperm TERMINAL FLOWER1 (TFL 1) genes. Sci Rep 7:14830. https://doi.org/10.1038/s41598-017-13645-0

14. Gelli M, Konda AR, Liu K, Zhang C, Clemente TE, Holding DR, Dweikat M (2017) Validation of QTL mapping and transcriptome profiling for identification of candidate genes associated with nitrogen stress tolerance in sorghum. BMC Plant Biol 17:123. https://doi.org/10.1186/ s12870-017-1064-9

15. Habib A, Powell JJ, Stiller J, Liu M, Shabala S, Zhou MX, Gardiner DM, Liu CJ (2018) A multiple near isogenic line (multi-NIL) RNA-seq approach to identify candidate genes underpinning QTL. Theor Appl Genet 131:613-624. https://doi.org/10.1007/s00122-017- 3023-0

16. Hempel FD, Welch DR, Feldman LJ (2000) Floral induction and determination: where is flowering controlled? Trends Plant Sci 5:17-21. https://doi.org/10.1016/s1360-1385(99) 01511-3

17. Huang NC, Jane WN, Chen J, Yu TS (2012) Arabidopsis thaliana CENTRORADIALIS homologue (ATC) acts systemically to inhibit floral initiation in Arabidopsis. Plant J 72:175-184. https://doi.org/10.1111/j.1365-313X.2012.05076.x

18. Huang CH, Zhang J, Zhou DH, Huang YT, Su L, Yang GL, Luo WL, Chen ZQ, Wang H, Guo T (2021) Identification and candidate gene screening of $q C I R 9.1$, a novel QTL associated with anther culturability in rice (Oryza sativa L.). Theor Appl Genet 134:2091-2111. https://doi.org/10.1007/s00122-021-03808-z 
19. Huang SN, Liu ZY, Yao RP, Li DY, Feng H (2015) Comparative transcriptome analysis of the petal degeneration mutant pdm in Chinese cabbage (Brassica campestris ssp. pekinensis) using RNAseq. Mol Genet Genomics 290(5):1833-1847. https://doi.org/10.1007/s00438 -015-1041-7

20. Jeong SY, Ahn H, Ryu J, Oh Y, Sivanandhan G, Won KH, Park, Young D, Kim J, Kim H, Lim YP, Kim SG (2019) Generation of early-flowering Chinese cabbage (Brassica rapa spp. pekinensis) through CRISPR/Cas9-mediated genome editing. Plant Biotechnol Rep 13:491- 499. https://doi.org/10.1007/s11816-019-00566-9

21. Jiang YF, Habib A, Zheng L, Zhou XM, Wei YM, Zheng YL, Liu CJ (2019) Development of tightly linked markers and identification of candidate genes for Fusarium crown rot resistance in barely by exploiting a near-isogenic line-derived population. Theor Appl Genet 132:217-225. https://doi.org/10.1007/s00122-018-3209-0

22. Kakizaki T, Kato T, Fukino N, Ishida M, Hatakeyama K, Matsumoto S (2011) Identification of quantitative trait loci controlling late bolting in Chinese cabbage (Brassica rapa L.) parental line Nou 6 gou. Breed Sci 61: 151-159. https://doi.org/10.1270/jsbbs.61.151

23. Kaur S, Atri C, Akhatar J, Mittal M, Kaur R, Banga SS (2021) Genetics of days to flowering, maturity and plant height in natural and derived forms of Brassica rapa L. Theor Appl Genet 134:473-487. https://doi.org/10.1007/s00122-020-03707-9

24. Kaneko-Suzuki M, Kurihara-Ishikawa R, Okushita-Terakawa C, Kojima C, Nagano-Fujiwara M, Ohki I, Tsuji H, Shimamoto K, Taoka KI (2018) TFL1-Like proteins in rice antagonize rice FT-like protein in inflorescence development by competition for complex formation with 14-3-3 and FD. Plant Cell Physiol 59:458-468. https://doi.org/10.1093/pcp/pcy021

25. Keurentjes JJ, Bentsink L, Alonso-Blanco C, Hanhart CJ, Blankestijn-De Vries H, Effgen S, Vreugdenhil D, and Koornneef M (2007) Development of a near-isogenic line population of Arabidopsis thaliana and comparison of mapping power with a recombinant inbred line population. Genetics 175:891905. https://doi.org/10.1534/genetics.106.066423

26. Li F, Kitashiba H, Inaba K, and Nishio T (2009a) A Brassica rapa linkage map of EST-based SNP markers for identification of candidate genes controlling flowering time and leaf morphological traits. DNA Res 16:311-323. https://doi.org/10.1093/dnares/dsp020

27. Li GL, Li F, Zhang SF, Zhang H, Zhang SJ, Sun RF (2019) Cloning and fucntion analysis of a novel flowering time regulatory gene BraELF6 s in Brassica rapa. Scientia Hortic 248:126-131. https://doi.org/10.1016/j.scienta.2019.01.009

28. Li H, Handsaker B, Wysoker A, Fennell T, Ruan J, Homer N, Marth G, Abecasis G, Durbin R, and 1000 Genome Project Data Processing Subgroup (2009b) The Sequence Alignment/Map format and SAMtools. Bioinformatics 25:2078-2079. https://doi.org/10.1093/ bioinformatics/btp352

29. Li XH, Wu M, Liu GY, Pei WF, Zhai HH, Yu JW, Zhang JF, Yu SX (2017) Identification of candidate genes for fiber length quantitaitive trait loci through RNA-Seq and linkage and physical mapping in cotton. BMC Genomics 18:427. https://doi.org/10.1186/s12864- 017-3812-5 
30. Li XN, Wang WK, Wang Z, Li KN, Lim YP, Piao ZY (2015) Construction of chromosome segment substitution lines enables QTL mapping for flowering and morphological traits in Brassica rapa. Frontiers in Plant Sci 6:432. https://doi.org/10.3389/fpls.2015.00432

31. Liu C, Thong Z, Yu H (2009) Coming into bloom: the specification of floral meristems. Development 136:3379-3391. https://doi.org/10.1242/dev.033076

32. Liu D, Teng Z, Kong J, Liu X, Wang W, Zhang X, Zhai T, Deng X, Wang J, Zeng J, Xiao Y, Guo K, Zhang J, Liu D, Wang W, and Zhang Z (2018) Natural variation in a CENTRORADIALIS homolog contributed to cluster fruiting and early maturity in cotton. BMC plant Biol 18: 286. https://doi.org/10.1186/s12870-018-1518-8

33. Liu XM, Bi B, Xu X, Li BH, Tian SM, Wang JP, Zhang H, Wang GQ, Han YJ, McElroy JS (2019) Rapid identification of a candidate nicosulfuron sensitivity gene (Nss) in maize (Zea mays L.) via combining bulked segregant analysis and RNA-seq. Theor Appl Genet 132:1351-1361. https://doi.org/10.1007/s00122-019-03282-8

34. Liu YT, Li CY, Shi XX, Feng Hui, Wang YG (2016) Identification of QTLs with additive, epistatic, and QTL $\times$ environment interaction effects for the bolting trait in Brassica rapa L. Euphytica 210:427-439. https://doi.org/10.1007/s10681-016-1710-6

35. Livak KJ, Schmittgen TD (2001) Analysis of relative gene expression data using real-time quantitative PCR and the $2^{-\triangle \Delta C t}$ method. Methods 25:402-408. https://doi.org/10.1006/meth. 2001.1262

36. Lou P, Zhao JJ, Kim JS, Shen SX, Carpio DP, Song XF, Jin M, Vreugdenhil D, Wang XW, Koornneer M, Bonnema G (2007) Quantitative trait loci for flowering time and morphological traits in multiple populations of Brassica rapa. J Exp Bot 58:4005-4016. https://doi.org/10.1093/jxb/erm255

37. Lou P, Xie Q, Xu X, Edwards CE, Brock MT, Weinig C, McClung CR (2011) Genetic architecture of the circadian clock and flowering time in Brassica rapa. Theor Appl Genet 123:397-409. https://doi.org/10.1007/s00122-011-1592-x

38. Lu H, Lin T, Klein J, Wang S, Qi J, Zhou Q, Sun J, Zhang Z, Weng Y, and Huang S (2014) QTL-seq identifies an early flowering QTL located near Flowering Locus $T$ in cucumber. Theor Appl Genet 127:1491-1499. https://doi.org/10.1007/s00122-014-2313-z

39. McKenna A, Hanna M, Banks E, Sivachenko A, Cibulskis K, Kernytsky A, Garimella K, Altshuler D, Gabriel S, Daly M, DePristo MA (2010) The Genome Analysis Toolkit: a MapReduce framework for analyzing next-generation DNA sequencing data. Genome Res 20:1297-1303. https://doi.org/10.1101/gr.107524.110

40. Murray MG, Thompson WF (1980) Rapid isolation of high molecular weight plant DNA. Nucleic Acids Res 8:4321-4325. https://doi.org/10.1093/nar/8.19.4321

41. Mimida N, Goto K, Kobayashi Y, Araki T, Ahn JH, Weigel D, Murata M, Motoyoshi F, Sakamoto W (2001) Functional divergence of the TFL 1-like gene family in Arabidopsis revealed by characterization of a novel homologue. Genes Cells 6:327-336. https://doi.org/10.1046/ j.13652443.2001.00425.x 
42. Nadean JH, Frankel WN (2000) The roads from phenotypic variation to gene discovery: mutagenesis versus QTLs. Nat Genet 25:381. https://doi.org/10.1038/78051

43. Narasimhan V, Danecek P, Scally A, Xue Y, Tyler-Smith C, and Durbin R (2016) BCFtools/RoH: a hidden Markov model approach for detecting autozygosity from next-generation sequencing data. Bioinformatics 32:1749-1751. https://doi.org/10.1093/bioinformatics/ btw044

44. Nesbitt TC, Tanksley SD (2002) Comparative sequencing in the genus Lycopersicon. Implications for the evolution of fruit size in the domestication of cultivated tomatoes. Genetics 162:365-379. https://doi.org/10.1093/genetics/162.1.365

45. Nguyen KL, Grondin A, Courtois B, Gantet P (2019) Next-generation sequencing accelerates crop gene discovery. Trends Plant Sci 24:263-274. https://doi.org/10.1016/j.tplants.2018.11.008

46. Nishioka M, Tamura K, Harada K (2005) Mapping of QTL for bolting time in Brassica rapa (syn.campestris) under different environmental conditions. Breed Sci 55:127-133. https://doi.org/10.1270/jsbbs.55.127

47. Osborn TC, Kole C, Parkin IA, Sharpe AG, Kuiper M, Lydiate, DJ, and Trick M (1997) Comparison of flowering time genes in Brassica rapa, B. napus and Arabidopsis thaliana. Genetics 146:1123-1129. https://doi.org/10.1093/genetics/146.3.1123

48. Park M, Lee JH, Han K, Jang S, Han J, Lim JH, Jung JW, Kang BC (2019) A major QTL and candidate genes for capsaicinoid biosynthesis in the pericarp of Capsicum chinense revelaed using QTL-seq and RNA-seq. Theor Appl Genet 132:515-529. https://doi.org/10.1007/ s00122-018-3238-8

49. Pnueli L, Carmel L, Hareven D, Gutfinger T, Alvarez J, Ganal M, Zamir D, Lifschitz E (1998) The SELFPRUNING gene of tomato regulates vegetative to reproductive switching of sympodial meristems and is the ortholog of CEN and TFL 1. Development 125:1979-1989. https://doi.org/10.1242/dev.125.11.1979

50. Ratcliffe OJ, Amaya I, Vincent CA, Rothstein S, Carpenter R, Coen ES, Bradley DJ (1998) A common mechanism controls the life cycle and architecture of plants. Development 125:1609-1615. https://doi.org/10.1242/dev.125.9.1609

51. Schubert M, Lindgreen S, and Orlando L (2016) AdapterRemoval v2: rapid adapter trimming, identification, and read merging. BMC Res notes 9:88. https://doi.org/10.1186/ s13104-016-1900-2

52. Shu JS, Liu YM, Zhang LL, Li ZS, Fang ZY, Yang LM, Zhuang M, Zhang YY, Lv HH (2018) QTL-seq for rapid identification of candidate genes for flowering time in broccoli $\times$ cabbage. Theor Appl Genet 131:917-928. https://doi.org/10.1007/s00122-017-3047-5

53. Su A, Song W, Xing J, Zhao Y, Zhang R, Li C, Duan M, Luo M, Shi Z, Zhao J (2016) Identification of genes potentially associated with the fertility instability of S-type cytoplasmic male sterility in maize via bulked segregant RNA-Seq. PLoS ONE 11:e0163489.

https://doi.org/10.1371/journal.pone.0163489

54. Su TB, Wang WH, Li PR, Zhang B, Li P, Xin XY, Sun HH, Yu YJ, Zhang DS, Zhao XY, Wen CL, Zhou G, Wang YT, Zheng HK, Yu SC, Zhang FL (2018) A genomic variation map provides insights into the 
genetic basis of spring Chinese cabbage (Brassica rapa ssp. pekinensis) selection. Mol Plant 11:1360-1376. https://doi.org/10.1016/j.molp.2018.08.006

55. Su TB, Wang WH, Li PR, Xin XY, Yu YJ, Zhao XY, Zhang DS, Yu SC, and Zhang FL (2021) Natural variations of BrHISN2 provide a genetic basis for growth-flavour trade-off in different Brassica rapa subspecies. New Phytol 231: 2186-2199. https://doi.org/10.1111/ nph.17515

56. Silva Lda C, Wang S, Zeng ZB (2012) Composite interval mapping and multiple interval mapping: procedures and guidelines for using windows QTL cartographer. Methods Mol Biol 871:75-119. https://doi.org/10.1007/978-1-61779-785-9_6

57. Takagi H, Abe A, Yoshida K, Kosugi S, Natsume S, Mitsuoka C, Uemura A, Utsushi H, Tamiru M, Takuno S, Innan H, Cano L M, Kamoun S, and Terauchi, R (2013) QTL-seq: rapid mapping of quantitative trait loci in rice by whole genome resequencing of DNA from two bulked populations. Plant J 74:174-183. https://doi.org/10.1111/tpj.12105

58. Teutonico RA, Osborn TC (1995) Mapping loci controlling vernalization requirement in Brassica rapa. Theor Appl Genet 91:1279-1283. https://doi.org/10.1007/BF00220941

59. Van der Auwera GA, Carneiro MO, Hartl C, Poplin R, Del Angel G, Levy-Moonshine A, Jordan T, Shakir K, Roazen D, Thibault J, Banks E, Garimella KV, Altshuler D, Gabriel S, and DePristo MA (2013) From FastQ data to high confidence variant calls: the Genome Analysis Toolkit best practices pipeline. Curr protoc Bioinformatics 43:11.10.1-11.10.33. https://doi.org/10.1002/0471250953.bi1110s43

60. Wang K, Li M, Hakonarson H (2010) ANNOVAR: Functional annotation of genetic variants from highthroughput sequencing data. Nucleic Acids Research 38:e164. https://doi.org/10.1093/ nar/gkq603

61. Wang TY, Hou LT, Jian HJ, Di FF, Li JN, Liu LZ (2018a) Combined QTL mapping, physiological and transcriptomic analyses to identify candidate genes involved in Brassica napus seed aging. Theor Appl Genet 293:1421-1435. https://doi.org/10.1007/s00438-018-1468-8

62. Wang YG, Zhang L, Ji XH, Yan JF, Liu YT, Lv XX, and Feng H (2014) Mapping of quantitative trait loci for the bolting trait in Brassica rapa under vernalizing conditions. GMR 13:3927-3939. https://doi.org/10.4238/2014.May.23.3

63. Wang YG, Wang XS, Wang X, Zhao QN, Feng H (2018b) Construction of chromosome segment substitution lines of Chinese cabbage (Brassica rapa L. ssp. pekinensis) in the background of $\mathrm{RcBr}$ (B. rapa L. ssp. dichotoma) and characterization of segments representing the bolting trait. Mol Breeding 38:35. https://doi.org/10.1007/s11032-018-0794-1

64. Wen J, Jiang F, Weng Y, Sun M, Shi X, Zhou Y, Yu L, and Wu Z (2019) Identification of heat-tolerance QTLs and high-temperature stress-responsive genes through conventional QTL mapping, QTL-seq and RNA-seq in tomato. BMC plant Biol 19:398. https://doi.org/ 10.1186/s12870-019-2008-3

65. Wellmer F, and Riechmann JL (2010) Gene networks controlling the initiation of flower development. Trends Genet 26: 519-527. https://doi.org/10.1016/j.tig.2010.09.001

66. Wu J, Wei KY, Cheng F, Li SK, Wang Q, Zhao JJ, Bonnema G, Wang XW (2012) A naturally occurring InDel variation in BraA.FLC.b (BrFLC2) associated with flowering time variation in Brassica rapa. BMC Plant Biol 12:151. https://doi.org/10.1186/1471-2229-12-151 
67. Xi X, Wei K, Gao B, Liu J, Liang J, Cheng F, Wang X, Wu J (2018) BrFLC5: a weak regulator of flowering time in Brassica rapa. Theor Appl Genet 131:2107-2116. https://doi.org/ 10.1007/s00122018-3139-x

68. Xiao D, Zhao JJ, Hou XL, Basnet RK, Carpio DPD, Zhang NW, Lin K, Cheng F, Wang XW, Bonnema G (2013) The Brassica rapa FLC homologue FLC2 is a key regulator of flowering time, identified through transcriptional co-expression networks. J Exp Bot 64(14): 4503-4516. https://doi.org/10.1093/jxb/ert264

69. Xiao D, Shen HR, Zhao JJ, Wei YP, Liu DR, Hou XL, Bonnema G (2019) Genetic dissection of flowering time in Brassica rapa responses to temperature and photoperiod. Plant Sci 280:110-119. https://doi.org/10.1016/j.plantsci.2018.10.027

70. Xiao YN, Thatcher S, Wang M, Wang TT, Beatty M, Hayes GZ, Li L, Li JS, Li BL, Yang XH (2016) Transcriptome analysia of near-isogenic lines provides molecular insights into starch biosynthesis in maize kernel. J Integr Plant Biol 58:713-723. https://doi.org/10.1111/jipb. 12455

71. Yang R, Gao H, Wang X, Zhang J, Zeng ZB, and Wu R (2007) A semiparametric approach for composite functional mapping of dynamic quantitative traits. Genetics 177:1859-1870. https://doi.org/10.1534/genetics.107.077321

72. Yang SJ, Zhang B, Liu G, Hong BH, Xu JS, Chen X, Wang B, Wu ZK, Hou F, Yue XP, Wang J, Zhang QH, King GJ, Liu KD (2018) A comprehensive and precise set of intervarietal substitution linse to identify candidate genes and quantitative trait loci in oilseed rape (Brassica napus L). Theor Appl Genet 131:2117-2129. https://doi.org/10.1007/s00122-018-3140-4

73. Yano M (2001) Genetic and molecular dissection of naturally occurring variation. Curr Opin Plant Biol 4:130-135. https://doi.org/10.1016/s1369-5266(00)00148-5

74. Yuan YX, Wu J, Sun RF, Zhang XW, Xu DH, Bonnema G, Wang XW (2009) A naturally occurring splicing site mutation in the Brassica rapa FLC1 gene is associated with variation in flowering time. $J$ Exp Bot 60:1299-1308. https://doi.org/10.1093/jxb/erp010

75. Zhang HW, Wang X, Pan CQ, Li P, Liu YJ, Lu XD, Zhong WS, Li MQ, Han LQ, Li J, Wang PX, Li DD, Liu Y, Li Q, Yang F, Zhang YM, Wang GY, Li L (2019) QTG-seq accelerates QTL fine mapping through QTL partitioning and whole genome sequencing of bulked segregant samples. Mol Plant 12: 426-437. https://doi.org/10.1016/j.molp.2018.12.018

76. Zhang L, Cai X, Wu J, Liu M, Grob S, Cheng F, Liang J, Cai C, Liu Z, Liu B, Wang F, Li S, Liu F, Li X, Cheng L, Yang W, Li MH, Grossniklaus U, Zheng H, Wang X (2018a) Improved Brassica rapa reference genome by single-molecule sequencing and chromosome conformation capture technologies. Hortic Res 5:50. https://doi.org/10.1038/ s41438-018-0071-9

77. Zhang S, Jiao Z, Liu L, Wang K, Zhong D, Li S, Zhao T, Xu X, Cui X (2018b) Enhancer-promoter interaction of SELF PRUNING 5 G shapes photoperiod adaptation. Plant Physiol 178:1631-1642. https://doi.org/10.1104/pp.18.01137

78. Zhang X, Meng L, Liu B, Hu Y, Cheng F, Liang J, Aarts MG, Wang X, Wu J (2015) A transposon insertion in FLOWERING LOCUS T is associated with delayed flowering in Brassica rapa. Plant Sci 
241:211-20. https://doi.org/10.1016/j.plantsci.2015.10.007

79. Zhao JJ, Kulkarni V, Liu NN, Carpio DPD, Bucher J, Bonnema G (2010) BrFLC2 (FLOWERING LOCUS C) as a candidate gene for a vernalization response QTL in Brassica rapa. J Exp Bot 61:1817-1825. https://doi.org/10.1093/jxb/erq048

80. Zhu Y, Klasfeld S, Jeong CW, Jin R, Goto K, Yamaguchi N, Wagner D (2020) TERMINAL FLOWER 1-FD complex target genes and competition with FLOWERING LOCUS T. Nat Commun 11:5118. https://doi.org/10.1038/s41467-020-18782-1

81. Zou C, Wang PX, Xu YB (2016) Bulked sample analysis in genetics, genomics and crop improvement. Plant Biotechnol J 14:1941-1955. https://doi.org/10.1111/pbi.12559

\section{Tables}

Table 1. Phenotypic values for $\mathrm{RcBr}^{1}$ and CSSL16 under four environments

\begin{tabular}{|c|c|c|c|c|c|c|c|c|}
\hline Trait & DE5 (d & & DE10 ( & & FT (day & & $\mathrm{BI}$ & \\
\hline Environment & $\mathrm{RcBr}$ & CSSL16 & $\mathrm{RcBr}$ & CSSL16 & $\mathrm{RcBr}$ & CSSL16 & $\mathrm{RcBr}$ & CSSL16 \\
\hline E1 & $\begin{array}{l}40.21 \\
\pm 0.94\end{array}$ & $\begin{array}{l}52.36 \pm \\
1.35^{\star \star}\end{array}$ & $\begin{array}{l}49.33 \\
\pm 2.27\end{array}$ & $\begin{array}{l}61.58 \pm \\
2.18^{\star \star}\end{array}$ & $\begin{array}{l}75.47 \\
\pm 3.78\end{array}$ & $\begin{array}{l}98.19 \pm \\
2.07 \star \star\end{array}$ & $\begin{array}{l}7.00 \pm \\
0.00\end{array}$ & $\begin{array}{l}3.00 \pm \\
0.00 \star \star\end{array}$ \\
\hline E2 & $\begin{array}{l}35.00 \\
\pm 1.36\end{array}$ & $\begin{array}{l}41.71 \pm \\
1.20 * \star\end{array}$ & $\begin{array}{l}40.46 \\
\pm 1.24\end{array}$ & $\begin{array}{l}44.00 \pm \\
0.88^{\star \star}\end{array}$ & $\begin{array}{l}52.26 \\
\pm 1.27\end{array}$ & $\begin{array}{l}63.43 \pm \\
1.28^{\star \star}\end{array}$ & $\begin{array}{l}7.00 \pm \\
0.00\end{array}$ & $\begin{array}{l}5.00 \pm \\
0.00 \star \star\end{array}$ \\
\hline E3 & $\begin{array}{l}21.00 \\
\pm 1.91\end{array}$ & $\begin{array}{l}27.71 \pm \\
0.09 * \star\end{array}$ & $\begin{array}{l}25.64 \\
\pm 1.65\end{array}$ & $\begin{array}{l}33.23 \pm \\
0.44^{\star \star}\end{array}$ & $\begin{array}{l}44.21 \\
\pm 3.55\end{array}$ & $\begin{array}{l}61.69 \pm \\
3.88^{\star \star}\end{array}$ & $\begin{array}{l}7.00 \pm \\
0.00\end{array}$ & $\begin{array}{l}5.00 \pm \\
0.00^{\star \star}\end{array}$ \\
\hline E4 & $\begin{array}{l}19.23 \\
\pm 1.12\end{array}$ & $\begin{array}{l}24.32 \pm \\
0.65^{\star \star}\end{array}$ & $\begin{array}{l}23.24 \\
\pm 1.73\end{array}$ & $\begin{array}{l}29.96 \pm \\
2.04^{\star \star}\end{array}$ & $\begin{array}{l}38.78 \\
\pm 2.78\end{array}$ & $\begin{array}{l}51.55 \pm \\
3.37^{\star \star}\end{array}$ & $\begin{array}{l}7.00 \pm \\
0.00\end{array}$ & $\begin{array}{l}5.00 \pm \\
0.00 \star \star\end{array}$ \\
\hline
\end{tabular}

E1-E4 are four different environment as same as Table S1. Data are presented as means \pm standard errors. significant levels: ${ }^{*}<0.05,{ }^{\star \star} P<0.01^{\prime}$

${ }^{1}$ Abbreviations: $\mathrm{RcBr}$, rapid-cycling inbred line of $B$. rapa; CSSL16, chromosome segment substitution line 16 ; DE5/DE10, days to reach a 5/10-cm-high elongated floral stalk; FT, flowering time; $\mathrm{BI}$, bolting index.

\section{Figures}

\section{Figure 1}

Sequence variations between $\mathrm{RcBr}$ and CSSL16 detected using whole-genome resequencing. Distribution of SNP-EDs and INDEL-EDs screened on chromosomes based on whole-genome resequencing. The $x$-axis 
represents the $10 \mathrm{~B}$. rapa chromosomes and the $y$-axis shows the ED values of the filtered SNP-EDs and INDEL-EDs. a The distribution of SNP-EDs. b The distribution of INDEL-EDs

\section{Figure 2}

Phenotypic characterization of $\mathrm{RcBr}$ and CSSL16. a The early flowering line 'RcBr' (left) and late flowering line 'CSSL16' (right) grown under natural conditions. b Column diagram evaluating the flowering time of the two lines in four different environments (E1, E2, E3 and E4). Data presented are means with SD ( $n=30$ plants). Scale bars $=5 \mathrm{~cm}, * \star$ Significant at $\mathrm{P}<0.01$

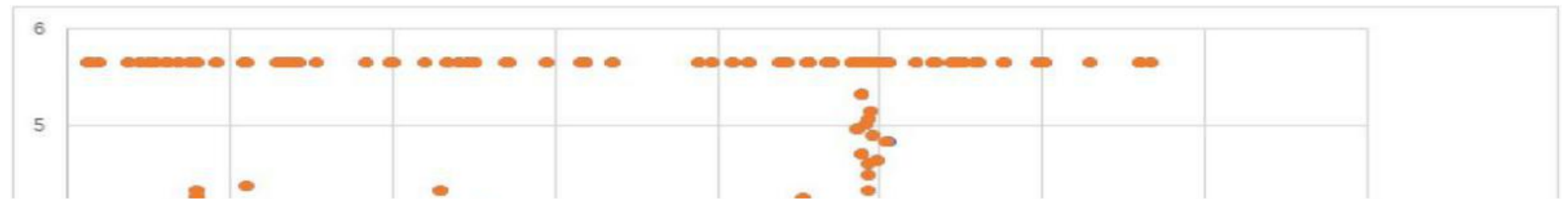

\section{Figure 3}

The distribution of the ED5 value of differential SNPs on Brassica rapa chromosomes according to BSRSeq analysis. BSR-Seq-based distribution of SNPs on chromosomes. The x-axis shows the $10 \mathrm{~B}$. rapa chromosomes and the $y$-axis shows the ED5 values of the filtered SNPs, the horizontal line is the threshold of the top $1 \%$

\section{Figure 4}

Fine mapping of qFT7.1 a Graphic representation of the genotype of chromosome A07 encompassing qFT7.1. Sequence variations of chromosome A07 between the two parental lines detected by whole genome re-sequencing. The orange region represents the confidence interval of qFT7.1 identified by BSRseq. b Traditional QTL mapping was preformed to validate the QTL qFT7.1. Physical map of the qFT7.1 region on chromosome A07. Traditional QTL analysis was used to detect qFT7.1, which was preliminary located between marker InDel714 and InDel716. The position of markers is shown on the $x$-axis and the LOD value is shown on the y-axis. The LOD value of qFT7. 1 was 32.2 , which explained the rate $39.9 \%$ of 
the phenotypic variation. c Fine mapping of qFT7.1. The phenotype and genotype of the ten homozygous recombinant groups and the two parental lines ( $\mathrm{RcBr}$ and CSSL16) used for fine mapping. The marker genotypes of RcBr are shown as black bars and those of CSSL16 are shown as white bars, DE5, DE10, and FT data appear as means \pm SD. Significant differences for the traits of the recombinant compared with those of the parents are indicated using superscript letters $(a, b)$. Student's t-test was used to distinguish significant difference at $P<0.01$. $d$ Structure of the BraA07g018240.3C coding region. Whole genome re-sequencing and cloning detected sequence variation of BraA07g018240.3C between the two parental lines, the black regions represent exons and the straight lines represent intron, two base variation mutations were identified in first and second exons

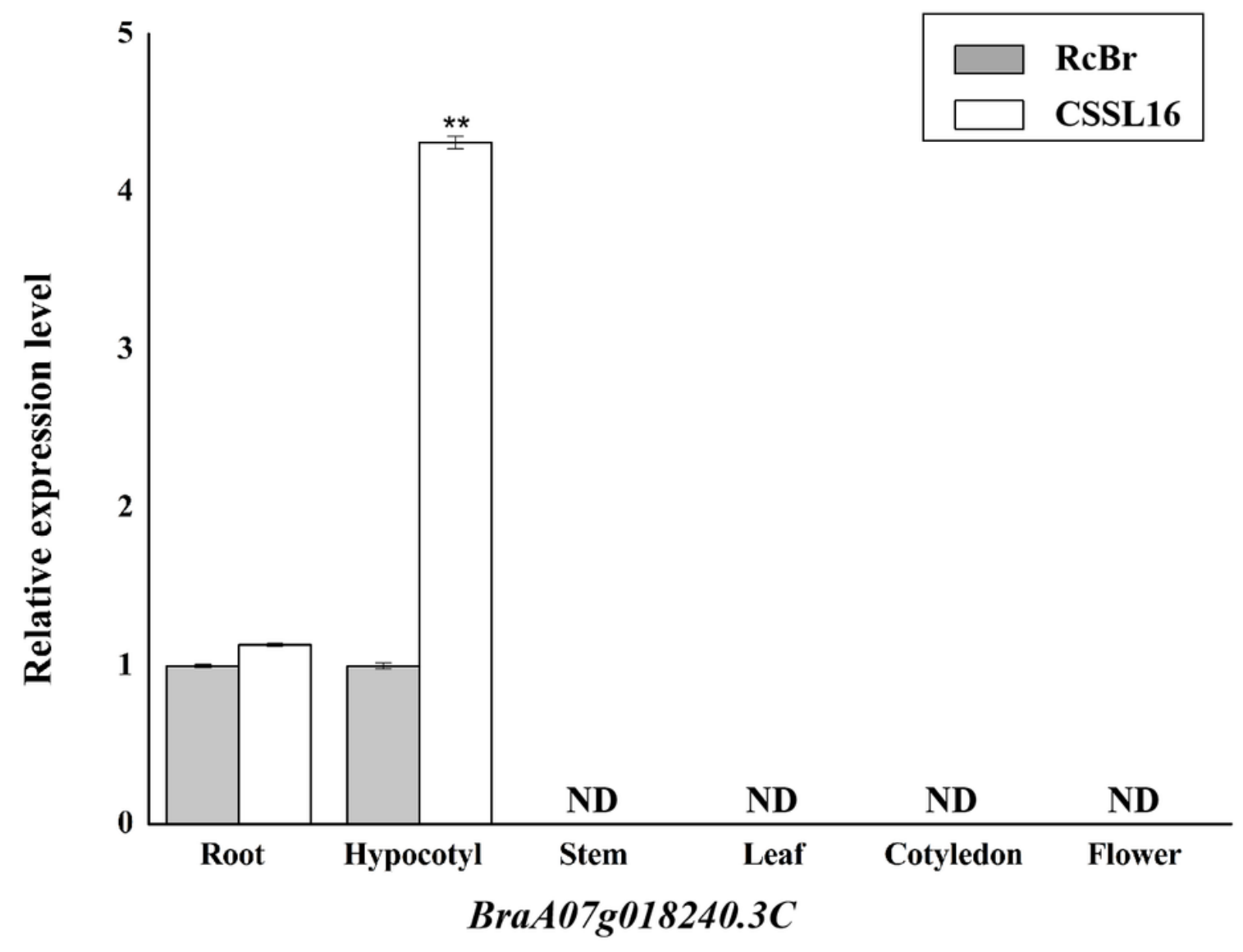

Figure 5

Expression patterns of BraA07g018240.3C in plant tissue BraA07g018240.3C expression in the root, hypocotyl, stem, leaf, cotyledon and flower, assessed using qRT-PCR. BraA07g018240.3C was especially expressed in the root and hypocotyl, and showed a significant difference in the hypocotyl. Error bars represent the standard errors from three replications, $\mathrm{P}<0.01$ 

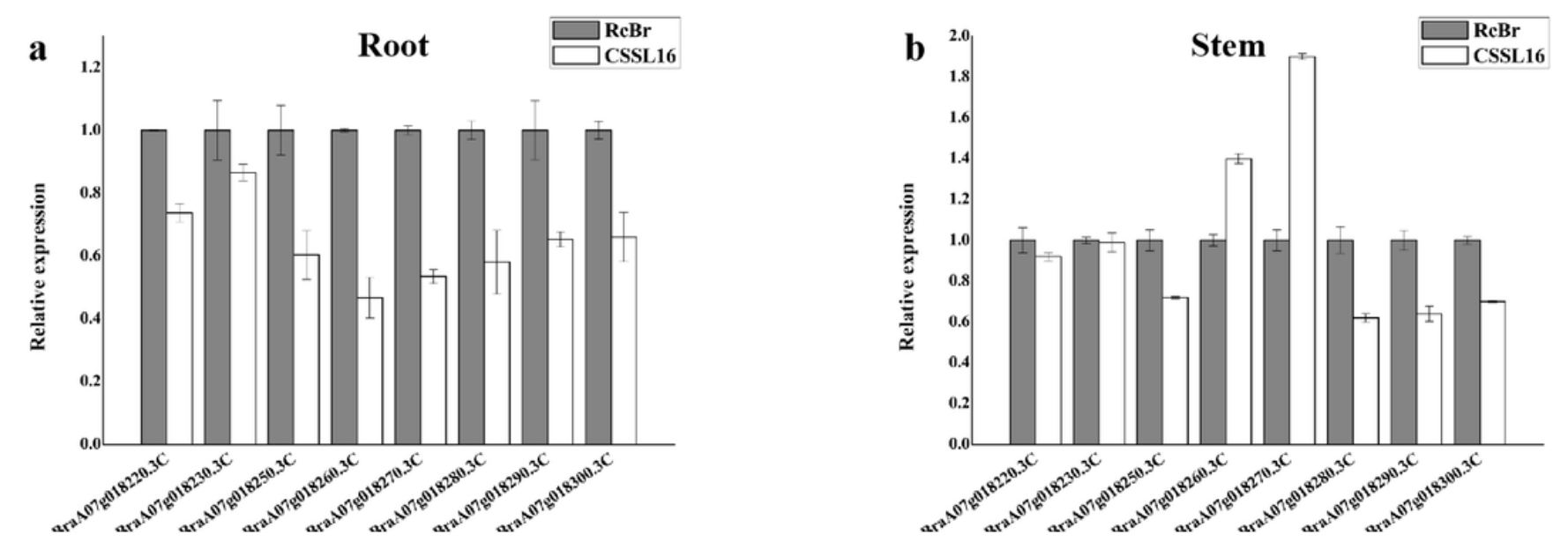

Figure 6

Expression level of eight genes in the candidate region Expression level of eight genes in (a) the root, (b) stem, (c) leaf and (d) flower determined using qRT-PCR. The expression levels of BraA07g018270.3C and BraA07g018300.3C showed significant differences in flower. Error bars represent the standard errors from three replications, $\mathrm{P}<0.01$ 

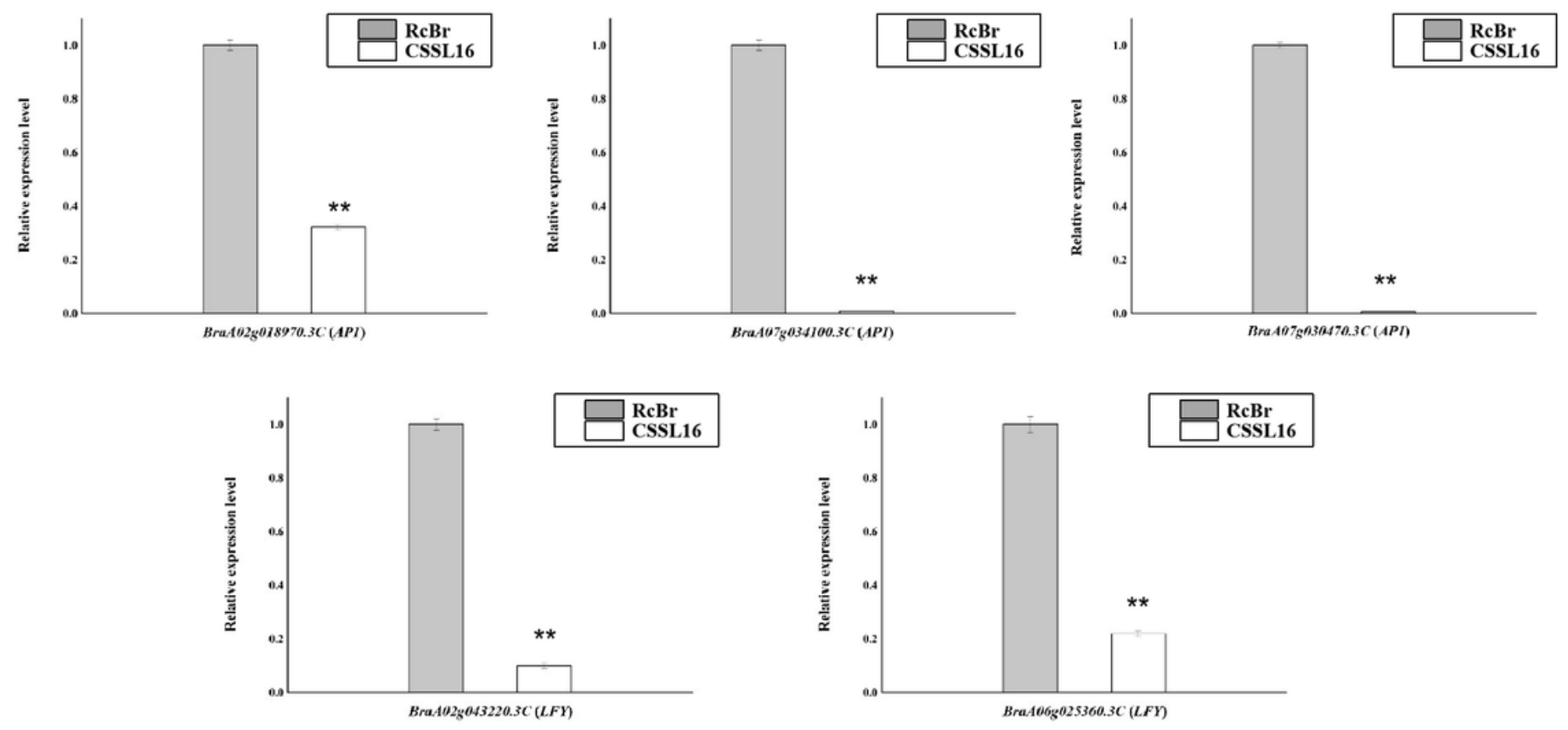

Figure 7

Expression level of an ATC-related genes in the flowering stage Expression of AP1 and LFY genes in B. rapa according to flowering time. The expression levels of AP1 (BraA02g018970.3C, BraA07g034100.3C, BraA07g030470.3C) and LFY genes (BraA02g043200.3C, BraA06g025360.3C) in 'RcBr' and 'CSSL16'. Error bars represent the standard errors from three replications, $\mathrm{P}<0.01$

\section{Supplementary Files}

This is a list of supplementary files associated with this preprint. Click to download.

- Figs1.tif

- Tables1.doc

- TableS2.xIsx

- Tables3.xls 